\title{
Eixo de Anatomia Radiológica do Curso de Medicina da Escola Bahiana de Medicina e Saúde Pública: Um Relato de Experiência
}

\author{
Rinaldo Antunes Barros ${ }^{1 *}$, Carolina Freitas Lins ${ }^{1}$, Marta Silva Menezes ${ }^{1}$, Beatriz Teixeira \\ Costa $^{2}$, Guilherme de Oliveira Dahia², Fernando Azevedo Medrado Junior ${ }^{2}$ \\ 'Docentes da Escola Bahiana de Medicina e Saúde Pública \\ ${ }^{2}$ Discentes da Escola Bahiana de Medicina e Saúde Pública \\ * Autor para correspondência: rinaldobarros@bahiana.edu.br
}

\section{RESUMO}

O avanço das técnicas de exames de imagem levou o diagnóstico clínico a um novo patamar na Medicina moderna, tornando-as indispensáveis para todos os médicos. A fim de que estejam aptos a avaliar adequadamente as imagens adquiridas, além de serem necessários conhecimentos em radiologia, é fundamental que os acadêmicos de Medicina se habituem com a anatomia normal vista através dos exames de imagem. Assim, é necessário que a matriz curricular contemple adequadamente essa necessidade através de estratégias pedagógicas eficazes. Tendo em vista tal diretriz, o ensino da Anatomia Radiológica na Escola Bahiana de Medicina e Saúde Pública (EBMSP) é integrado à abordagem da Anatomia Topográfica no componente curricular Introdução à Técnica Operatória (quatro horas de carga horária semanal). Duas horas semanais são reservadas à Anatomia Radiológica e são divididas em uma hora de discussão clínico-radiológica com docente e uma hora de atividade prática com monitores. As outras duas horas são destinadas ao outro eixo, Anatomia Topográfica. Semanalmente, uma região topográfica é abordada. No eixo Anatomia Topográfica, a região é descrita; na discussão clínico-radiológica, um caso clínico é conduzido, abordando o papel dos exames de imagem na investigação diagnóstica, e a atividade prática com os monitores é dividida em três estações (salas) que abordam a identificação de estruturas em três exames de imagem diferentes. Observa-se assim que a implementação do eixo Anatomia Radiológica na EBMSP é uma ação que visa à adequação da matriz curricular às demandas profissionais ao potencializar a formação acadêmica e o aprendizado do aluno desde os primeiros anos da graduação, no que diz respeito à habilidade de interpretação de exames de imagem.

Palavras-chave: Educação Médica; Anatomia Radiológica; Currículo Médico.

\begin{abstract}
The advancement of imaging techniques led clinical diagnosis to a new level in modern Medicine, making them indispensable for all physicians. In order to be able to properly interpret acquired images, in addition to knowledge in radiology, it is essential that medical students become accustomed to the normal anatomy seen through imaging examinations. Thus, it is necessary that the curriculum matrix adequately addresses this need through effective pedagogical strategies. In view of this guideline, the teaching of the Radiological Anatomy at the Bahiana School of Medicine and Public Health (EBMSP) is integrated to the Topographic Anatomy approach in the Introduction to the Operative Technique curriculum (four hours of weekly hours). Two hours a week are assigned to Radiological Anatomy and are divided into one hour of clinical-radiological discussion with lecturer and one hour of practical activity with monitors. The other two hours are assigned to the other component, Topographic Anatomy. A topographic region is addressed weekly. In the topographic anatomy component, the region is described, in the clinical-radiological discussion a clinical case is conducted addressing the role of imaging tests in the diagnostic investigation and the practical activity with the monitors is divided into three stations (rooms) that deal with the identification of structures in three different imaging tests. It is observed that the implementation of the Radiological Anatomy approach in the EBMSP aims to adapt the curricular matrix to the professional demands by potentiating the academic formation and learning of student from the first years of graduation, regarding the ability to interpret imaging tests.
\end{abstract}

Keywords: Medical Education; Radiological Anatomy; Medical Curriculum. 


\section{Introdução}

A anatomia tem importância fundamental na matriz curricular dos cursos de Medicina; entretanto, existe um debate acerca dos métodos disponíveis para o ensino desse componente (DETTMER et al., 2015, pp. 260-8). A integração da radiologia com o ensino da anatomia promove maior satisfação dos alunos, em comparação com o método tradicional de ensino, bem como melhor sucesso na aprendizagem (MURPHY et al., 2015, pp. 510-7).

A fim de que os médicos estejam aptos a avaliar adequadamente as imagens adquiridas, além de serem necessários conhecimentos básicos em radiologia, é fundamental que, ainda enquanto acadêmicos de Medicina, habituem-se à anatomia normal vista através das diversas técnicas disponíveis (MITCHELL \& WILLIAMS, 2002, pp. 1070-2). A aquisição de habilidades de interpretação das principais alterações dos exames de imagem é também fundamental, pois torna o futuro profissional menos dependente do especialista, sobretudo em situações de emergência nas quais decisões precisam ser tomadas em tempo hábil (Idem, ibidem; GUNDERMAN, SIDDIQUI, HEITKAMP KIPFER, 2003, pp. 1239-42).

$\mathrm{O}$ avanço nas técnicas de exames de imagem levou o diagnóstico clínico a um novo patamar na Medicina moderna, tornando-as indispensáveis para todos os médicos (PASCUAL, CHHEM, WANG \& VUJNOVIC, 2011, pp. 319-25; GELEIJNS, BREATNACH, CANTERA \& DAMILAKIS, 2012, pp. 197-200). Além disso, a radiologia é um componente cada vez mais integrativo do ponto de vista da prática médica, já que virtualmente todas as especialidades obtêm informações diagnósticas fundamentais através dos exames de imagem (GUNDERMAN, SIDDIQUI, HEITKAMP KIPFER, 2003, pp. 1239-42; PHILLIPS, SMITH \& STRAUS, 2013, pp. 297-305).

A Universidade College Cork, na Irlanda, utiliza um modelo que integra anatomia e radiologia desde 2006. Os estudantes do primeiro ano têm atividades que englobam aulas teóricas, tutoriais, sessões de aprendizado em grupos pequenos sobre radiologia, combinados com visualização de peças cadavéricas, dissecção, além de softwares para identificação de estruturas anatômicas. Nessa instituição, o ensino da radiologia na graduação médica é percebido como algo importante pela maioria dos estudantes de Medicina. O ensino integrado da anatomia com a radiologia permite familiaridade com os métodos radiológicos, bem como melhor identificação de estruturas anatômicas nos exames de imagem. Além disso, os estudantes preferem sessões interativas combinando anatomia e radiologia a aulas teóricas de anatomia e abordagem de peças cadavéricas em laboratório (PHILLIPS, SMITH \& STRAUS, 2013, pp. 297-305).

Um método particularmente útil para integrar ensino de anatomia e radiologia é a ultrassonografia (USG). Em trabalho prévio, 91\% dos anatomistas consultados opinaram que a USG reforça conceitos anatômicos e 95\%, que a utilização do método na graduação médica fortalece a compreensão de correlações anátomo-clínicas (ROYER, 2016, pp. 453-467).

A matriz curricular dos cursos de Medicina deve, portanto, contemplar adequadamente essa necessidade, implementando estratégias pedagógicas eficazes para alcançar o melhor resultado possível, bem como ofertando carga horária suficiente para tal aprendizado (BRANSTETTER, FAIX, HUMPHREY \& SCHUMANN, 2007, pp. 9-14). Nesse contexto, o presente relato de experiência tem como objetivo discutir práticas, princípios pedagógicos, estratégias e técnicas empregados na implementação do eixo de Anatomia Radiológica do curso de Medicina da Escola Bahiana de Medicina e Saúde Pública (EBMSP).

\section{Relato de Experiência}

O eixo de Anatomia Radiológica do curso de Medicina da EBMSP foi implementado com o propósito primordial de promover o desenvolvimento de habilidades de identificação da anatomia humana por meio de exames de imagem, bem como habituar os discentes aos aspectos básicos da radiologia. Simultaneamente, familiariza os acadêmicos 
com a técnica de aquisição de imagens, com a natureza biofísica de cada exame, com a nomenclatura apropriada para a descrição dos achados em cada técnica e com a propedêutica radiológica. Os princípios que nortearam tal implementação, por sua vez, foram a abordagem transversal da radiologia, teórico-prática e integrada ao ensino da anatomia, perpassando pelos semestres do Ciclo Básico.

As atividades de Anatomia Radiológica são desenvolvidas ao longo dos três primeiros semestres do curso. No primeiro, realiza-se uma conferência de abertura do eixo, na qual são abordados os métodos de exame de imagem (Radiografia simples, Radiografia contrastada e ultrassonografia) e suas peculiaridades, como técnica básica de aquisição do exame, terminologia de descrição de achados, principais aplicações, vantagens e desvantagens. Nesse semestre e no subsequente (segundo), a Anatomia Radiológica integra o componente curricular Biomorfologia, que aborda a anatomia humana por sistemas através do método Aprendizado Baseado em Problemas (APB). Os sistemas são agrupados em módulos, com duração de seis semanas cada um, sendo que em cada semana há um caso clínico que ancora a discussão das ciências básicas morfológicas. Os exames de imagem são contemplados nos objetivos das discussões tutoriais, proporcionando contato semanal do estudante com a radiologia. Ainda nessa fase, ao final de cada módulo ocorre a revisão do conteúdo estudado, cujas atividades incluem uma problematização integrativa dos casos clínicos abordados pela docente radiologista, a fim de sistematizar e sedimentar o conhecimento adquirido nas semanas anteriores.

Finalizado o primeiro ano, o eixo passa a integrar o componente curricular Introdução à Técnica Operatória (ITO), que, além da Anatomia Radiológica, contempla o ensino de Anatomia Topográfica. Aborda-se uma região corporal por semana, em onze semanas. A estratégia de ensino radiológico nessa fase é também topográfica. Dessa forma, são abordadas as aplicações dos métodos de imagem em afecções-modelo que acometem a região da semana, bem como a anatomia topográfica nos exames. Apesar de não haver discussão em tutoria no componente curricular em questão, os discentes têm acesso, por meio da plataforma Moodle ${ }^{\circledR}$, no Ambiente Virtual de Aprendizagem (AVA), a um caso clínico do eixo Anatomia Radiológica na segunda-feira, que serve de base para a discussão em encontro presencial que ocorre na sexta-feira. Tal encontro é dividido em dois momentos, que acontecem concomitantemente, em sistema de rodízio: um seminário teórico interativo ministrado por um docente especialista e estações práticas de exames de imagem ministradas por monitores (Figura 1).

No seminário teórico interativo, que dura sessenta minutos, os estudantes se dispõem em cinco mesas redondas dotadas de computadores nos quais uma apresentação de diapositivos, contendo cinco questões relacionadas ao caso da semana, é exibida. O objetivo do seminário é

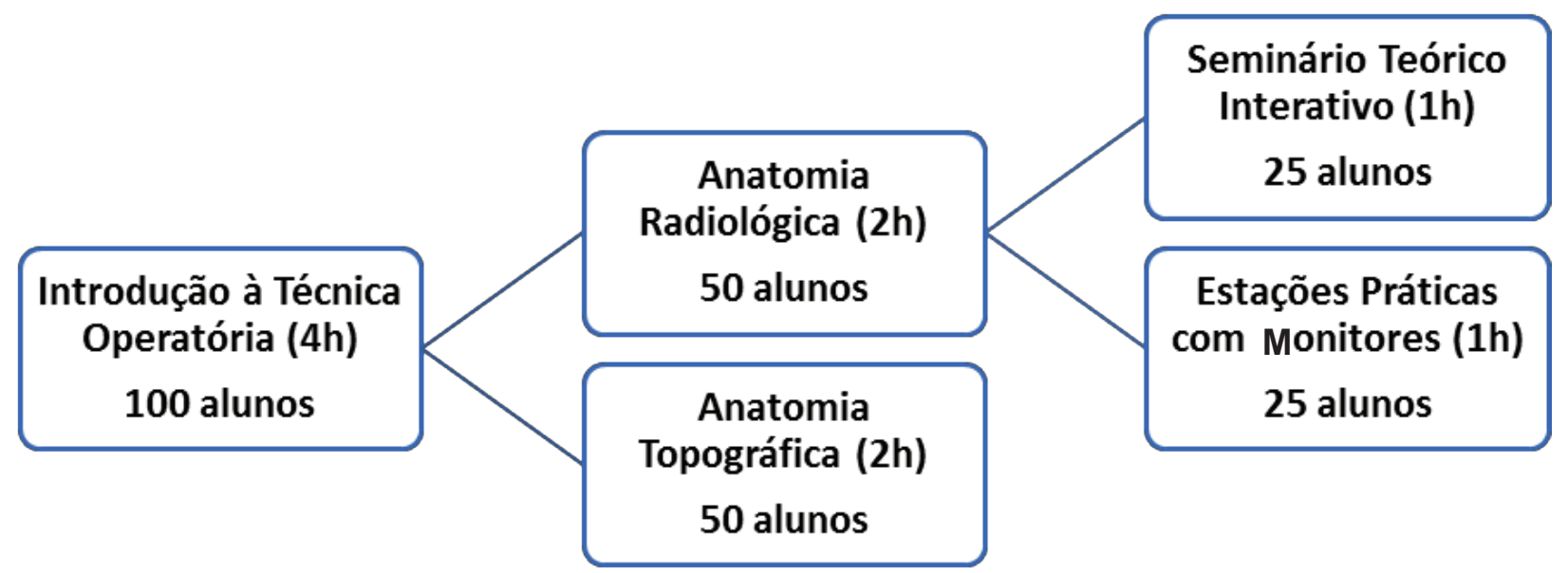

Figura 1 - Distribuição dos acadêmicos no componente curricular de Introdução à Técnica Operatória. 
abordar a radiologia aplicada à região, construindo em conjunto (docente-discente) respostas para o caso clínico da semana, além de extrapolar para outras doenças daquela região através de exemplos com o objetivo de enriquecer o aprendizado (Figura 2). Não obstante, realiza-se ainda o enfoque à propedêutica radiológica e à solicitação adequada do exame, traduzindo-se em questões como o raciocínio clínico que subsidia o pedido: as peculiaridades de cada solicitação (uso ou não de contraste, ultrassonografia com ou sem Doppler, por exemplo), forma de redigir o pedido adequadamente, dentre outras.

As estações práticas, por sua vez, são ministradas por monitores do Núcleo de Ensino e Pesquisa em Anatomia Radiológica (NEPAR) devidamente capacitados e têm o objetivo de facilitar a transposição do conhecimento anatômico para os exames de imagem. Em cada sexta-feira são três temas abordados nas estações práticas, cada um com duração de quinze minutos, também em sistema de rodízio, totalizando 45 minutos. É importante frisar que os discentes permanecem na mesma estação (sala) durante os 45 minutos, de modo que os monitores, cada um responsável por um tema, revezam-se ao término de cada uma, além de haver um monitor, com função de coordenador da semana, que esclarece dúvidas logísticas dos alunos e controla a duração das estações para garantir o funcionamento adequado das atividades (Figura 3), permitindo aos estudantes o contato com imagens radiológicas para

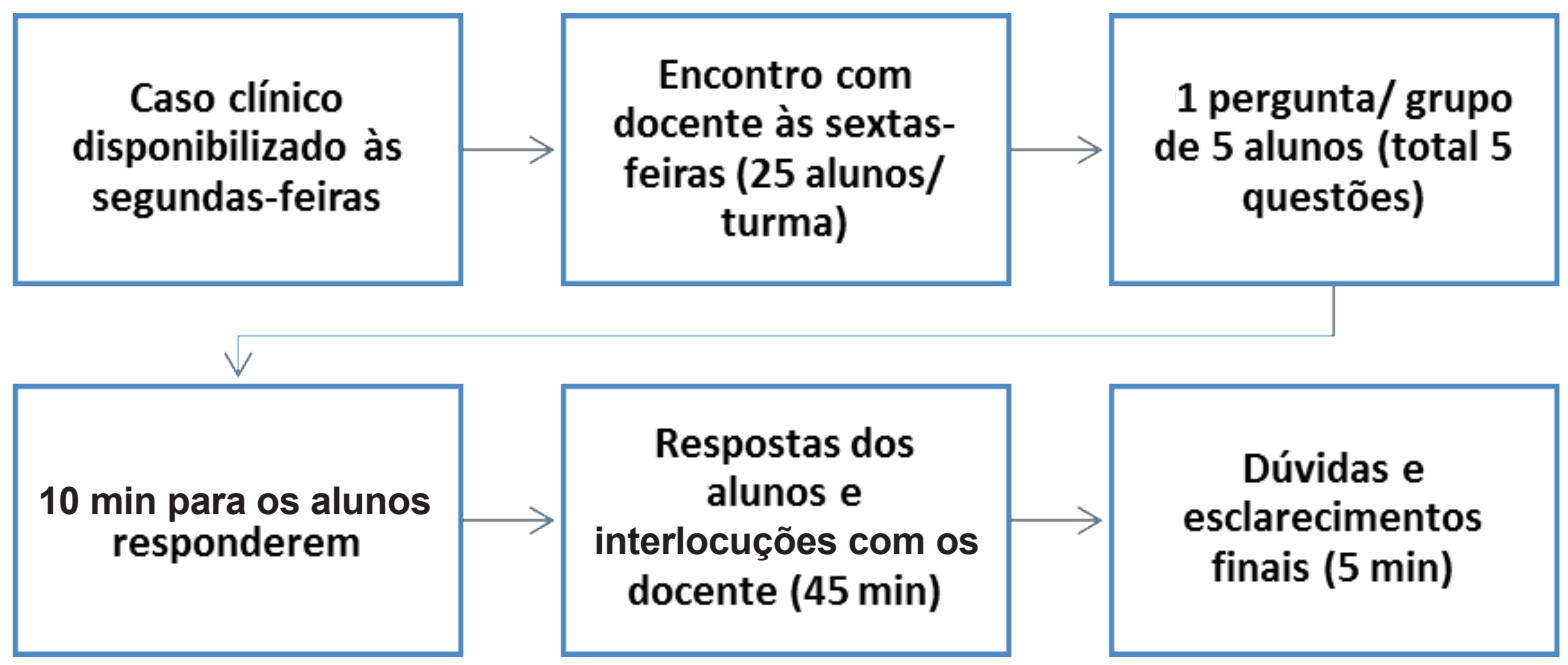

Figura 2 - Esquema demonstrativo das atividades no seminário teórico interativo realizadas pela docente especialista.

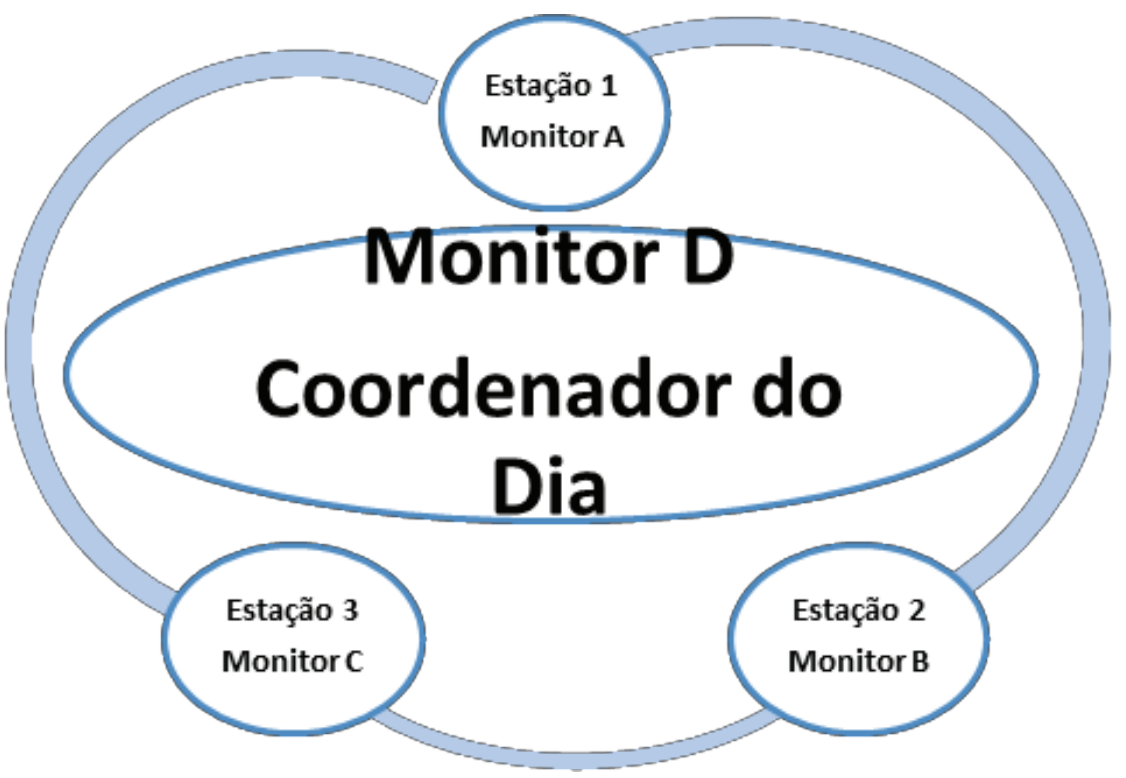

Figura 3 - Esquema demonstrando a distribuição dos monitores nas estações práticas. 
que eles façam as devidas correlações anatômicas.

Para fins de exemplificação, na semana de Cavidade Torácica, por exemplo, as atividades funcionam da seguinte maneira: os cem estudantes do terceiro semestre são divididos em dois grupos de cinquenta (grupos A e B). Durante a primeira hora, o grupo $A$ tem atividades de Anatomia Topográfica e o grupo $B$ de Anatomia Radiológica. Os cinquenta discentes do grupo $B$ são divididos em dois grupos de 25 (B1 e B2): B1 tem, primeiro, o seminário teórico interativo, e os alunos de B2, divididos em três grupos (dois grupos com oito integrantes e um com nove), começam pelas estações práticas. Nessa referida semana, ocorrem as estações de Radiografia de tórax, Tomografia de tórax e Ultrassonografia/Ressonância de tórax. $\mathrm{Na}$ segunda hora, os alunos de $B 2$ vão para o seminário e os de $B 1$, para as estações (Figura 4). Depois, os alunos do grupo $B$ vão para as atividades de Anatomia Topográfica e os do grupo $A$, para Anatomia Radiológica. O ciclo descrito para o grupo $B$ nas duas primeiras horas se repete da mesma forma para o grupo $A$, totalizando quatro horas semanais de carga horária do componente curricular ITO.

\section{Discussão}

Durante semestres mais avançados do curso de Medicina da EBMSP existem componentes curriculares nos quais os alunos desenvolvem atividades práticas voltadas para a interpretação de exames de imagem. Entretanto, vem sendo demonstrado que a exposição prévia aos conhecimentos sobre métodos radiológicos básicos, como ultrassonografia (MILES, 2005, pp. 742-5; GESINE et al., 2013, p. 1), tomografia computadorizada e ressonância magnética (BIASUTTO, 2010, pp. 112-114), bem como o contato com recursos avançados como endoscopia virtual, abordagem funcional $/ \mathrm{molecu}$ lar e espectroscopia (DEETMER et al., 2013, pp. 838-843), durante os primeiros anos da graduação, proporcionam novas possibilidades para a inclusão desses exames de imagem no ensino de componentes fundamentais como a anatomia.

Apesar de essas novas ferramentas estarem disponíveis para o incremento do ensino da anatomia, o conhecimento de alguns estudantes ainda é considerado muito baixo, principalmente no campo da anatomia clínica. Essa realidade fica evidente quando conceitos básicos de disposição anatômica

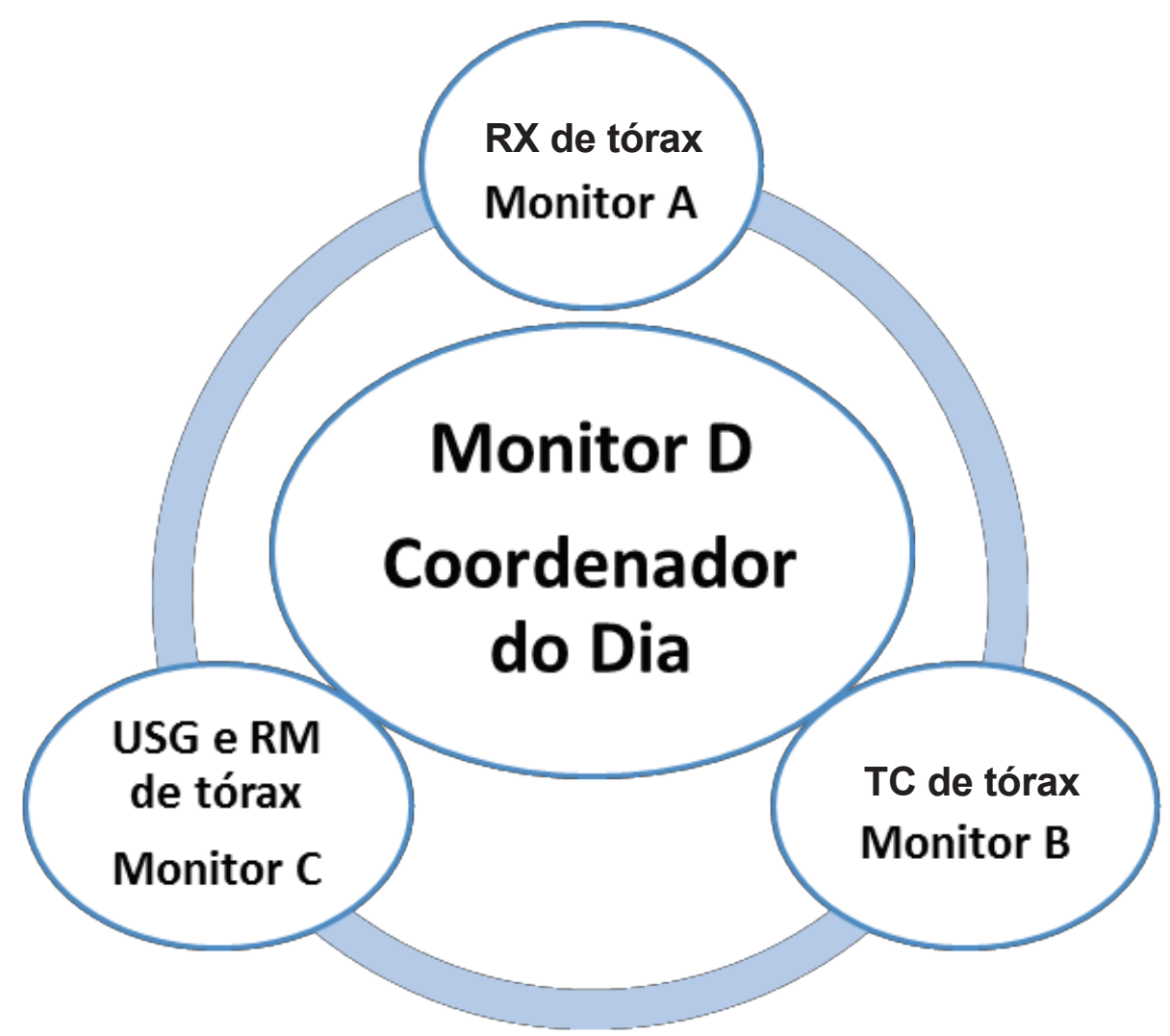

Figura 4 - Esquema demonstrativo do exemplo das atividades nas estações práticas da monitoria. 
são esquecidos no momento de interpretar um exame no contexto hospitalar, ou marcos anatômicos soam estranhos quando o laudo de um exame de imagem é exposto. Falta a habilidade de correlacionar a prática médica com o alicerce teórico inicial do curso de Medicina (MERAL SAVRAN et al., 2015, pp. 568-75). Esse despreparo tem sido observado sobretudo em alunos de instituições que não aderem a métodos inovadores e, assim, teriam maior evasão dos estudantes no componente curricular da anatomia (GALENUS, 2014, pp. 62-8).

Existem possíveis aperfeiçoamentos a serem realizados no eixo Anatomia Radiológica, como o desenvolvimento de sessões práticas nas quais os alunos possam realizar exames, a exemplo de estações de ultrassonografia. Nas determinadas estações, o aluno seria capaz de examinar diversas regiões anatômicas, bem como aprender técnicas do exame na prática com o auxílio de um docente.

Médicos e estudantes de Medicina devem possuir conhecimentos básicos da anatomia, bem como da radiologia, já que técnicas de imagens transversais se tornaram rotina não só para a realização de diagnóstico, mas também para o auxílio de procedimentos invasivos (LISIECKA et al., 2016, pp. 295-303). Nesse contexto, a implementação do eixo Anatomia Radiológica na EBMSP é uma ação que visa à adequação da matriz curricular às demandas profissionais, ao potencializar a formação acadêmica e o aprendizado do aluno desde os primeiros anos da graduação acerca de ambos os componentes.

\section{Referências Bibliográficas}

BIASUTTO, S. N. "Anatomical Knowledge and Diagnostic Images in Medical Education". Revista Argentina de Anatomía Clínica, vol. 2, n. 3, September 2010, pp. 112-114.

BRANSTETTER, B. F,; FAIX, L. E.; HUMPHREY, A. L. \& SCHUMANN, J. B. "Preclinical Medical Student Training in Radiology: the Effect of Early Exposure". AfR Am $\mathcal{F}$ Roentgenol, vol. 188, n. 1, 2007, pp. 9-14.

DEETMER, S.; WEIDEMANN, J.; FISCHER, V. \& WACKER, F. K. "Radiological Anatomy - Evaluation of Integrative Education in Radiology". Fortschr Röntgenstr, vol. 185, 2013, pp. 838-843.
; ANTOCH, G.

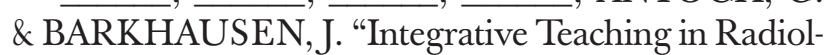
ogy - A Survey"/"Integrative Lehre in der Radiologie". RoFo: Fortschritte auf dem Gebiete der Rontgenstrahlen und der Nuklearmedizin, vol. 187, n. 4, Apr. 2015, pp. 260-8.

GALENUS, C. "Desafio Anatômico: Metodologia Capaz de Auxiliar no Aprendizado da Anatomia Humana". Anatomia. Medicina (Ribeirão Preto), vol. 47, n. 1, 2014, pp. 62-8.

GELEIJNS, J.; BREATNAGH, É.; CANTERA, A. C. \& DAMILAKIS, J. "Core Curriculum for Medical Physicists in Radiology. Recommendations from an EFOMP / ESR Working Group". Insights into Imaging, vol. 3, n. 3, 2012, pp. 197-200.

GESINE, G.; SGHRAMEK, R.; STOEVESANDT, D.; REISING, A.; KIELSTEIN, J. T. \& HISS, M. "Imaging in Anatomy: a Comparison of Imaging Techniques in Embalmed Human Cadavers". BMC Med Educ [Internet]. BMC Medical Education, vol. 13, n. 1, 2013, p. 1. Available from: BMC Medical Education.

GUNDERMAN, R. B.; SIDDIQUI, A. R.; HEITKAMP, D. E. \& KIPFER, H. D. "Opinion: The Vital Role of Radiology in the Medical School Curriculum". Am $\mathcal{F}$ Roentgenol, vol. 180, n. 5, 2003, pp. 1239-1242.

LISIECKA, J.; PIETRZYK, Ł.; CZEKAJLO, M.; TORRES, A.; STA, G.J. \& ARANCIBIA, C. U. "Bridging the Gap between Basic and Clinical Sciences: A Description of a Radiological Anatomy Course". Anatomical Sciences Education, vol. 9, n. 3, 2016, pp. 295-303.

MERAL SAVRAN, M.; TRANUM-JENSEN, J.; FROST CLEMENTSEN, P.; HASTRUP SVENDSEN, J.; HOLST PEDERSEN, J. \& SEIER POULSEN, S. "Are Medical Students Being Taught Anatomy in a Way that Best Prepares Them to Be a Physician?" Clin Anat [Internet], vol. 28, n. 5, 2015, pp. 568-75.

MILES, K. A. Diagnostic Imaging in Undergraduate Medical Education: An Expanding Role. Clin Radiol., vol. 60, n. 7, 2005, pp. 742-5.

MITCHELL, B. S. \& WILLIAMS, J. E. "Trends in Radiological Anatomy Teaching in the U.K. and Ireland". Clin. Radiol., vol. 57, n. 12, 2002, pp. 1070-2.

MURPHY, K. P.; CRUSH, L.; O'MALLEY, E.; DALY, F. E.; TWOMEY, M. \& O'TUATHAIGH, C. M. P. "Medical Student Perceptions of Radiology Use in Anatomy Teaching". Anat Sci Educ., vol. 8, n. 6, 2015, pp. 510-7.

PASGUAL, T. N. B.; CHHEM, R.; WANG, S. C. \& VUJNOVIC, S. "Undergraduate Radiology Education in the Era of Dynamism in Medical Curriculum: An Educational Perspective". Eur F Radiol., vol. 78, n. 3, 2011, pp. 319-25. 
PHILLIPS, A. W.; SMITH, S. G. \& STRAUS, C. M. "The Role of Radiology in Preclinical Anatomy. A Critical Review of the Past, Present, and Future". Acad Radiol [Internet]. Elsevier Ltd., vol. 20, n. 3, 2013, pp. 297-305. Available from: http://dx.doi.org/10.1016/j. acra.2012.10.005.
ROYER, D. F. "The Role of Ultrasound in Graduate Anatomy Education: Current State of Integration in the United States and Faculty Perceptions". Anatomical Sciences Education, vol. 9, n. 5, 2016, pp. 453-467.

Publicado em 31/03/2017. 